\title{
Instrumentos de apoio financeiro para parques tecnológicos: a experiência de Minas Gerais
}

Inaiara Cóser Sobrinho ${ }^{1}$

Eduardo Gonçalves ${ }^{2}$

Resumo: No contexto da Economia do Aprendizado, o aprendizado decorre da interação entre os agentes para obtenção de novos conhecimentos e tecnologias, sendo que a inovação passa a ser o diferencial competitivo de empresas e regiões. Entre os habitats de estímulo à inovação espalhados pelo mundo, inclusive pelo Brasil, estão inseridos os chamados Parques Tecnológicos que consistem em um empreendimento complexo que necessita de um volumoso aporte financeiro. Esse trabalho descreve as fontes de recursos disponíveis para a consolidação desse empreendimento, com base na experiência de Minas Gerais. Dentre os resultados, destaca-se a importância da política pública do Estado de Minas Gerais mesmo na ausência de um programa nacional que suporte a criação desses habitats.

Palavras-chave: inovação; parques tecnológicos; apoio financeiro.

1 Economista, Universidade Federal de Juiz de Fora. E-mail: inaiaracoser@gmail.com

2 Prof. Adjunto da Faculdade de Economia, Pesquisador do CNPq, Universidade Federal de Juiz de Fora eduardo.gonçalves@ufjf.edu.br 


\title{
Instruments of Financial Support for Technology Parks: the experience of Minas \\ Gerais
}

\begin{abstract}
Based on the theoretical framework of the Learning Economy, the learning stems from the interaction between agents to obtain new knowledge and technologies and the innovative process becomes competitive advantage for firms and regions. The Technology Parks are one of the main habitats to support the creation of new technologies around the world which consist of a complex undertaking that requires a large financial commitment. This paper aims to describe the available sources of funds for the consolidation of this venture, based on the experience of the State of Minas Gerais. The main results highlightthe importance of the public support in Minas Gerais, although some guidelines to create a huge nationwide program are outlined.
\end{abstract}

Keywords: innovation; technology parks; financial support.

\section{Introdução}

Na dinâmica de desenvolvimento da economia mundial, o fator inovação torna-se fundamental para a criação de um parque produtivo nacional competitivo. Neste contexto, as empresas intensivas em tecnologia atuam como aditivos e catalisadores dos demais setores da economia e constituem a base da nova sociedade do conhecimento (ABDI/ANPROTEC 2007).

O conhecimento, acoplado à inovação, pode ser apontado como fator central na discussão sobre o aumento da competitividade das empresas e o desenvolvimento das nações, regiões, setores, empresas e até indivíduos (Cassiolato \& Lastres 2000). Diante disso, as várias esferas de governo têm incentivado cada vez mais a interação entre instituições de ensino e empresas, por meio de políticas públicas de apoio à inovação.

Essa interação entre os três setores - instituições de ensino, empresas e governo - pode ser representada pela perspectiva de Sistema Nacional de Inovação (SNI). Concebido por Lundvall (1988), o SNI apresenta a influência das instituições externas sobre as atividades inovadoras de empresas e outros atores, enfatizando a importância da transferência e difusão de ideias, conhecimentos e informações. Johnson e Lundvall (2005) citam a importância dos sistemas de inovação tanto como instrumento analítico quanto como guia para a elaboração de políticas. Este trabalho aborda o modelo de SNI Triple Helix.

Diante do exposto, o desafio se dá em converter produção científica em produtos de alto valor agregado para as empresas, aumentando a competitivi- 
dade e, consequentemente, gerando emprego de qualidade, bem-estar social e impostos. Nesse sentido, autores como Ichikawa e Santos (2001) veem a interação universidade/empresa como instrumento para criação de nichos de inovação tecnológica, empregos e prosperidade social.

Dentre as diversas ações necessárias para promoção da inovação, estão inseridos os habitats de inovação. Um dos exemplos de habitats de inovação são os Parques Tecnológicos (PqTs), objeto de estudo desse artigo.

Os PqTs são considerados ambientes desenvolvidos especialmente para abrigar empresas com alto valor agregado e para a promoção da interação entre conhecimento científico e produção. Tais empresas podem ser oriundas do processo de incubação ou de spin-offs.

Por se tratar de um ambiente complexo, o empreendimento PqT necessita de um volumoso aporte financeiro para sua implantação e sustentabilidade. Além de uma estrutura especialmente planejada, este empreendimento necessita de recursos humanos altamente capacitados, por isso é comum que se localizem próximos às universidades e centros de pesquisa (Steiner et al. 2008). Os autores afirmam que essa proximidade gera sinergias e oportunidades. Destarte, pode-se considerar a obtenção de recursos um dos principais entraves à realização desse empreendimento.

Diante do exposto, há a necessidade de levantar informações sobre linhas de financiamentos de PqTs, tanto na sua implementação quanto na sua sustentabilidade. Com este propósito, os próximos capítulos abordam a interação entre universidade e empresa e posteriormente as políticas públicas de incentivo à inovação no Brasil e, também, políticas específicas do Estado de Minas Gerais. Dessa forma, este trabalho se propõe a fazer um levantamento dos instrumentos de apoio financeiro disponíveis para implantação e operacionalização de PqTs no Estado de Minas Gerais.

\section{O SNI e A Interação Universidade-Empresa}

O conceito de SNI ressalta as diferenças no ritmo em que os países exploram as possibilidades oferecidas pelo hiato tecnológico que se abre, especialmente em tempos de mudança do paradigma técnico-econômico ou das trajetórias tecnológicas (Freeman \& Perez 1988). Essas diferenças são dependentes da capacidade de cada país de mobilizar recursos políticos e financeiros para transformar as estruturas tecnológicas, institucionais e econômicas que englobam seu SNI.

O SNI consiste no fluxo da tecnologia e da informação entre as pessoas, as empresas e as instituições, que são chave do processo inovativo. De acordo com a teoria de sistema da inovação, o desenvolvimento da inovação e da tecnologia é resultado de um jogo complexo dos relacionamentos entre atores 
do sistema, que inclui empresas, universidades e governo (OECD 1997). Além disso, no SNI devem ser incluídos os padrões evolutivos vindos de tendências históricas e culturais que influenciam os mecanismos de política pública (Lundvall 1992). Nesse sistema a interação entre universidades e empresas ciência e tecnologia - pode ser considerada como estratégica. Essa interação tem mão dupla, a ciência às vezes lidera, às vezes segue a inovação industrial. Podem-se destacar cinco funções das universidades nos sistemas de inovação: i) Formação de pessoal em geral; ii) Formação de pessoal capacitado para a geração de spin-offs; iii) Geração de conhecimento; iv) Interação com firmas estabelecidas e; $v$ ) Canal para absorção de conhecimento gerado nos centros mais avançados. (Bernardes \& Albuquerque 2003).

Uma importante característica dos SNI é que eles mudam ao longo do tempo, geralmente de forma gradual, mas às vezes acentuadamente (Nelson 1988). Isso ocorre por se tratar de um sistema dependente do aprendizado. Além de ser um sistema social, consiste em um sistema altamente dinâmico. Mais do que uma simples soma das atividades empresariais de um país, um SNI consiste no resultado das sinergias que surgem a partir da interação e dinâmica entre os atores econômicos (Mothe \& Paquet 1998).

Dentre os principais modelos de SNI, será abordado o modelo Triple Helix.

O modelo da Triple Helix insere-se no contexto da segunda revolução acadêmica, na qual as universidades repensam qual o seu papel nas relações com a sociedade. Além de ensino e pesquisa, a universidade incorpora uma terceira missão como um interveniente ativo no processo de desenvolvimento econômico através da criação de conhecimento científico e tecnológico e, consequentemente, inovação. A Triple Helix é essencialmente um modelo de análise da inovação numa economia baseada no conhecimento e este modelo auxilia no entendimento de como o sistema de inovação é baseado em expectativas (Leydesdorff \& Etzkowitz 1998).

Etzkowitz e Leydesdorff (1997) consideram a relação universidade-indústriagoverno como uma tríplice hélice de evolução das redes de comunicação.

A palavra hélice pode ser definida como aparelho de propulsão, de tração ou de sustentação, formada de pás implantadas sobre um eixo central e apresentando uma superfície helicoidal. De forma análoga, pode-se entender que a estrutura universidade-indústria-governo, para Etzkowitz e Leydesdorff (1997), corresponde ao aparelho de sustentação da economia, ou seja, propulsor do desenvolvimento.

A abordagem Triple Helix compreende a interação entre as três hélices, como forma de identificar e lidar com mudanças profundas no mundo econômico, institucional e intelectual, para uma sociedade organizada na base do conhecimento. Essas interações ocorrem em muitos níveis e resultam em (Etzkowitz 2002): $i$ ) transformações internas em cada hélice, tais como o 
desenvolvimento de relações laterais entre as empresas, através de alianças estratégicas ou de um pressuposto de uma missão de desenvolvimento econômico por parte das universidades; ii) a influência de organizações de uma esfera na outra, por exemplo, o papel do governo federal em instituir uma política indireta industrial no Bayh-Dole Act de 1980; iii) a criação de uma nova sobreposição de redes trilaterais e organizações a partir da interação entre as três hélices, formada com o propósito de chegar com novas ideias e formatos para o desenvolvimento de alta tecnologia.

Desta forma podemos dizer que a Triple Helix atua e se desenvolve por meio de três estágios distintos (Etzkiwitz e Leydesdorff 2000; Etzkowitz 2002; Leydesdorff e Etzkowitz 1998):

1) Modelo Triple Helix I (Figura 1), o Estado envolve o meio acadêmico e a indústria e dirige as relações entre eles.

2) Modelo Triple Helix II (Figura 2), as esferas institucionais são separadas, possuem fronteiras fortes que as dividem e as relações entre elas são altamente circunscritas.

3) Modelo Triple Helix III (Figura 3) apresenta uma sobreposição das esferas institucionais que consiste numa infra estrutura de conhecimento, com cada um interagindo com o papel do outro e com organizações híbridas que emergem em relação às interfaces.

FIGURA O1 . MODELO TRIPLE HELIX I

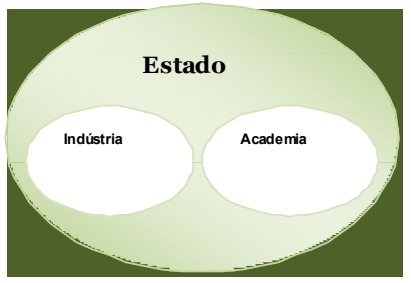

FONTE: Adaptado de Etzkowitz e Leydesdorff (2000: 111)
FIGURA O2 . MODELO TRIPLE HELIX II

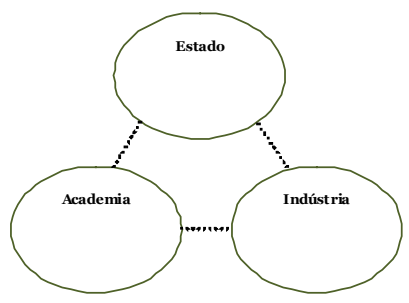

FONTE: Adaptado de Etzkowitz e Leydesdorff (2000: 111)

FIGURA O3 . MODELO TRIPLE HELIX III

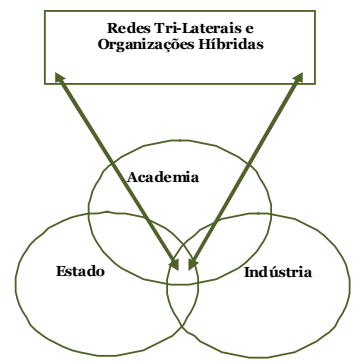

FONTE: Adaptado de Etzkowitz e Leydesdorff (2000: 111) 
Segundo Etzkowitz e Leydesdorff (2000), a maioria dos países e regiões tenta construir alguma forma de Triple Helix III, pois o objetivo comum é conceber um ambiente inovador que consiste em spin-off de universidades, iniciativas trilaterais do conhecimento, como base do desenvolvimento econômico, alianças estratégicas entre as empresas (grandes e pequenas, operando em diferentes áreas, e que operam em diferentes níveis de tecnologia), laboratórios governamentais e grupos de pesquisa acadêmica.

Triple Helix denota não apenas a relação da universidade, indústria e governo, mas também a transformação interna dentro de cada uma dessas esferas, este pode ser considerado um modelo analítico capaz de compreender o processo de inovação e de propor e implementar políticas públicas, especialmente em ciência, tecnologia e inovação com o objetivo de ampliar e apoiar a interação entre os diferentes atores que compõem o modelo (Etzkowitz e Leydesdorff 2000).

\subsection{Mecanismos de transferência de tecnologia}

Diante desse novo contexto, em que as universidades assumem os novos papéis de criação de conhecimento científico e tecnológico destacados na seção anterior, há evidências crescentes de que as universidades estão se tornando empreendedoras. Dentro dessa função, as novas empresas criadas a partir das universidades constituem-se como mecanismo de comercialização de resultados de pesquisas universitárias (Siegel et al. 2007).

Existem três tipos de fluxos entre universidade e indústria: quando o produto é originado na universidade e desenvolvido por uma firma existente; quando o produto é desenvolvido dentro da empresa e melhorado através de conhecimentos acadêmicos e: quando o produto é criado dentro da universidade, e o inventor cria uma empresa para comercializá-lo (spin-off acadêmica) (Etzkowitz 1998).

Dentre estes, a geração de spin-offs acadêmicas aparece como um importante mecanismo de transferência de tecnologia das universidades para a sociedade e, cada vez mais, é incentivado pelo setor governamental.

Outro mecanismo que pode ser destacado como importante para a interação universidade-empresa são os habitats de inovação.

Para Ullah e Taylor (2005), as pequenas empresas, principalmente as de base tecnológica, possuem características especiais e precisam de ambientes especiais para crescer e florescer. Estas condições especiais podem estar presentes em PqTs e incubadoras de empresas. Logo, nascem os habitats de inovação, ambientes preparados para receberem empresas inovadoras.

Os habitats de inovação se apresentam de diferentes formas, “(...) podendo ser configurados como incubadoras de empresas, condomínios empresariais, 
parques e pólos tecnológicos ou ainda tecnópolis” (Zen et al. 2004, p. 1).

Com o propósito de aprofundar a compreensão sobre o tema será analisado o conceito e objetivo dos PqTs e os impactos regionais gerados por este empreendimento.

\subsubsection{Parques tecnológicos e seus impactos e seus impac- tos regionais}

O interesse por PqT se iniciou na Universidade de Stanford (Califórnia - Estados Unidos) que culminou na experiência chamada -Vale do Silício. Esta experiência influenciou inúmeros modelos mundiais de criação de espaços que estimulassem a interação entre a atividade acadêmica das universidades com a iniciativa privada (Zouain, 2003).

Com o propósito de criar oportunidades e evitar a evasão de cérebros locais, a criação de PqTs próximos às universidades e instituições de ensino é empregada como uma estratégia vital para o desenvolvimento de indústrias de alta tecnologia em muitos países (Yang et al. 2009).

PqTs têm como missão prover a "inteligência", a infraestrutura e os serviços necessários ao crescimento e fortalecimento das empresas intensivas em tecnologia (ABDI/ANPROTEC 2007). O segmento de PqTs pode e deve representar para a "indústria do conhecimento e inovação" o mesmo que as universidades significaram para o segmento de ensino.

Para Morais et al. (2006), a função precípua de um PqT é estimular a interação entre o empreendedor, a tecnologia, a informação, os recursos físicos e financeiros e a capacitação técnica e estratégica do empreendedor. Desta forma, cria-se um sistema de acumulação de conhecimento para geração de spin-offs e uma teia de apoios e parcerias.

A International Association of Science Parks - IASP (2002:1), define PqTs como sendo

uma organização ministrada por profissionais especializados, cujo principal objetivo é aumentar a riqueza de sua comunidade, promovendo a cultura da inovação e a competitividade de seus negócios associados e instituições baseadas em conhecimento. Para permitir que estas metas sejam alcançadas, o Parque Científico estimula e administra o fluxo de conhecimento e tecnologia entre universidades, instituições de P\&D, empresas e mercados; facilita a criação e crescimento de empresas baseadas em inovação por meio de mecanismos de incubação e processos de spin-off; e provê outros serviços sobre o valor agregado juntamente com espaço e instalação de alta qualidade. (IASP 2002:1) 
Além de toda infraestrutura preparada para receber empresas de base tecnológica, o agrupamento dessas empresas em um único espaço gera benefícios adicionais, tais como redes interempresas, articulação com universidades e instituições de pesquisa e spillover tecnológico (Yang et al. 2009).

Segundo estudo realizado pela ABDI/ANPROTEC (2007), nos últimos anos houve um crescimento acentuado do número de projetos de parques no Brasil. Este estudo aponta quatro razões para esta expansão:

1) Fortalecimento da consciência dos atores governamentais acerca da importância da inovação para o desenvolvimento sustentável e crescimento econômico do país;

2) Aumento significativo do número de empresas interessadas em se instalar em PqTs - empresas geradas ou graduadas em incubadoras, empresas multinacionais de tecnologia e empresas nacionais determinadas a fortalecer suas unidades de P\&D;

3) Experiência bem sucedida de outros países que estão investindo de forma consistente neste mecanismo;

4) Necessidade de governos estaduais e municipais identificarem novas estratégias de estimular o crescimento e direcionar o desenvolvimento de suas regiões.

PqTs possuem objetivos relacionados tanto a impactos sobre as empresas quanto sobre as regiões locais. Objetivos relativos às empresas incluem a facilitação da interação universidade-empresa, estímulo à formação de empresas de base tecnológica, atração de empresas envolvidas com tecnologias de ponta, e promoção de alianças estratégicas e redes. Objetivos relacionados ao impacto regional incluem o desenvolvimento econômico, criação de emprego e melhoria da qualidade de vida local (Siegel et al., 2003).

Em que pese a importância de tais objetivos, há também casos em que a literatura aponta modestas contribuições dos PqTs sobre o desenvolvimento regional. Segundo Hilpert e Ruffieux (1991), a contribuição dos parques para o desenvolvimento regional depende das condições iniciais como firmas inovativas, infra-estrutura de pesquisa adequada etc. Além disso, muitos parques tornam-se grandes "elefantes brancos" por causa da ausência de uma estratégia adequada que defina seus objetivos ou porque estes objetivos não estão sintonizados com as reais necessidades e possibilidades da região (Lacave 1996; Martins 1996). Mais recentemente, alguns estudos apontam que os retornos da localização de uma empresa dentro de um parque são desprezíveis, como no caso do Reino Unido (SIEGEL et al., 2003).

Por outro lado, os PqTs parecem ser uma experiência bem sucedida em muitos países, tais como Suécia (Lindelof \& Losften 2002; Losften \& Lindelof 2002), China (Hu, 2007) e Taiwan (Yang et al., 2009). Neste último, ao realizarem 
um estudo que compara a produtividade de P\&D de novas empresas de base tecnológica localizadas dentro e fora de PqTs, os autores comprovaram que as empresas desenvolvidas dentro de PqTs investem de forma mais eficiente em inovação. Os ganhos de eficiência podem ser atribuídos ao apoio de políticas governamentais para empresas com esforços em P\&D, vantagem de localização, efeito clustering e externalidades de rede. $\mathrm{Na}$ China, $\mathrm{Hu}$ (2007) argumenta que o estabelecimento de PqTs como política de desenvolvimento regional tem conseguido alcançar o objetivo de aumentar o crescimento econômico nas regiões menos desenvolvidas. Na Suécia, Löfsten e Lindelöf (2002) constataram que os PqTs suecos cumprem um objetivo importante de política regional, pois empresas instaladas nos parques apresentaram melhores resultados no que tange à geração de emprego, além de apresentarem maior propensão a manterem vínculo com as universidades em relação a empresas fora dos parques.

Tudo isso revela que, embora haja um grau de importância variável de acordo com as condições regionais existentes, acreditamos que instrumentos como parques tecnológicos não são a solução para todos os problemas regionais, mas podem constituir importante elemento no processo de reestruturação econômica (Jowitt 1991). É importante destacar que os PqTs são apenas um dos diversos instrumentos de política pública de apoio à inovação e que as universidades, principalmente em países em desenvolvimento como o Brasil, estão começando a incorporar a missão de universidade empreendedora, elemento este importante para o sucesso de um PqT. A literatura mostra que na teoria e em muitos casos concretos o PqT é instrumento de desenvolvimento regional. Como afirma Yang et al. (2009), há a necessidade de se fazer um levantamento de quais são as condições críticas necessárias para a criação de um PqT bem sucedido, pois, se esta questão for elucidada, então o modo de desenvolvimento dos PqTs pode ser transplantado para os países em desenvolvimento, para ajudar no futuro desenvolvimento econômico dessas nações. Por se tratar de um instrumento de desenvolvimento, PqTs constituem “(...) mecanismos estratégicos no contexto de uma Política Pública mais abrangente e de caráter nacional/regional" (ABDI/ANPROTEC 2007: 9). Destarte, o empreendimento PqT deve contar com forte investimento estatal, pelo menos na sua implantação. Diante disso, há a necessidade de levantar informações sobre linhas de financiamentos de PqTs, tanto na sua implementação quanto na sua sustentabilidade. Com este propósito, o próximo capítulo aborda as políticas públicas de incentivo à inovação no Brasil e, também, políticas específicas do Estado de Minas Gerais.

\section{Políticas públicas de apoio à inovação}

O objetivo dessa seção é apresentar brevemente o aparato legal erigido no Brasil e no Estado de Minas Gerais para dar suporte à inovação tecnológica. 
No Brasil, a caracterização de sistemas nacionais de inovação se deu tardiamente. As reformas mais profundas, no que tange a políticas públicas de apoio à inovação, surgem no final da década de noventa. O apoio à CT\&I é constituído, principalmente, pelos Fundos Setoriais de Ciência e Tecnologia, pela "Lei de Inovação" e pela "Lei do Bem" (Morais 2008; Pacheco 2005).

Os Fundos Setoriais de Ciência e Tecnologia foram criados a partir de 1999 e constituem instrumentos de financiamento de projetos de pesquisa, desenvolvimento e inovação no Brasil. Para Morais (2008), os fundos têm como principais objetivos: garantir a ampliação e a estabilidade de recursos financeiros para P\&D; impulsionar os investimentos privados em pesquisa e inovação; fomentar parcerias entre as universidades, instituições de pesquisa e o setor produtivo ; e assegurar a continuidade dos investimentos em P\&D nos setores privatizados ou abertos aos investimentos privados na década de 1990. Para que os recursos aportados pelos fundos setoriais sejam alocados nos projetos de pesquisa, desenvolvimento e inovação há a atuação da Financiadora de Estudos e Projetos (Finep).

Segundo a FINEP (2010), existem atualmente no Brasil 16 Fundos Setoriais, sendo 14 relativos a setores específicos e dois transversais. ${ }^{3}$ Destes, um é voltado à interação universidade-empresa (Fundo Verde-Amarelo, FVA) e o outro é destinado a apoiar a melhoria da infraestrutura de Instituições de Ciência e Tecnologia (ICTs) (Infraestrutura). ${ }^{4}$

Em relação ao marco legal existente no Brasil, a lei federal 10.973 de 02/12/2004, conhecida como Lei de Inovação, regulamentada pelo decreto 5.563 de 11/10/2005, foi criada com o intuito de capacitar e alcançar a autonomia tecnológica e o desenvolvimento industrial do País. Dispõe sobre incentivos à inovação e à pesquisa científica e tecnológica no ambiente produtivo, além de apresentar apoio à construção de ambientes de inovação (BRASIL 2004). Essa lei envolve mudanças institucionais importantes no sentido de exigir maior agilidade e flexibilidade nas instituições públicas de pesquisa, abrindo novas oportunidades de cooperação com o setor produtivo (Pacheco 2005).

A Lei de Inovação representa um novo paradigma para a maior difusão do conhecimento gerado nas instituições de ensino e pesquisa em apoio a inovações no setor produtivo (Morais 2008). Além disso, o autor apresenta um amplo conjunto de ações, instituído pela Lei de Inovação, para o desenvolvimento

3 Ações Transversais são programas estratégicos do MCT que têm ênfase na Política Industrial, Tecnológica e de Comércio Exterior (PITCE) do Governo Federal e utilizam recursos de diversos Fundos Setoriais simultaneamente (FINEP 2010).

4 As receitas dos fundos proveem de diversas fontes: 1) parcela de royalties incidentes sobre a produção de petróleo e gás natural; 2) contribuições das empresas sobre os resultados da exploração de recursos naturais pertencentes à União, como mineração e energia elétrica; 3) 0,5\% do faturamento das empresas beneficiadas pela Lei de Informática; e 4) Contribuição de Intervenção no Domínio Econômico (Cide) incidente sobre as remessas ao exterior para pagamento de royalties, assistência técnica e serviços técnicos, com alíquota de $10 \%$ (Guimaraes 2006 apud Morais 2008). 
tecnológico: 1) criou condições legais para a formação de parcerias entre universidades, instituições privadas de C\&T sem fins lucrativos e empresas; 2) concedeu flexibilidade às instituições de ciência e tecnologia (ICT) públicas para participar de processos de inovação; 3) estabeleceu condições de trabalho mais flexíveis para os pesquisadores de ICT públicas; 4) criou modalidade de apoio financeiro por meio de subvenção econômica direta para empresas.

Posterior à Lei de Inovação, foi criada a lei de incentivos fiscais à inovação e exportação, também conhecida como Lei do Bem. A Lei no 11.196 de 21 de novembro de 2005, em seu Capítulo III, artigos 17 a 26, regulamentada pelo decreto 5.798 de 7 de junho de 2006, discorre que as pessoas jurídicas podem usufruir de incentivos fiscais, de forma automática, desde que realizem pesquisa tecnológica e desenvolvimento de inovação tecnológica.

No tocante aos incentivos à inovação, essa lei dá cumprimento à determinação da Lei 10.793, que estabelece que a União deve fomentar a inovação nas empresas mediante concessão de incentivos fiscais.

As legislações citadas proveram a base jurídica para a ampliação da presença do Estado no apoio à inovação, que vem atuando de forma mais eficaz junto ao setor produtivo. Além disso, a partir da aprovação e regulamentação da Lei de Inovação e da Lei do Bem se iniciou no Brasil o processo de subvenção econômica à inovação. Esta modalidade consiste na concessão de recursos financeiros de natureza não reembolsável para empresas que desenvolvam projetos de inovação estratégicos para o País de acordo com a política governamental (FINEP 2006). Com a criação da subvenção econômica, o governo diminui o risco tecnológico das empresas para inovar e para aumentar a capacidade inovativa do Brasil, assim como sua competitividade.

\subsection{Aparato legal de suporte à inovação em Mi-}

\section{nas Gerais}

Em consonância com o governo nacional, o Estado de Minas Gerais também vem realizando políticas públicas de incentivo à inovação. Para tal fim, foram criados a Lei Mineira de Inovação, o Fundo de Incentivo à Inovação Tecnológica (FIIT), além do Plano Mineiro de Desenvolvimento Integrado (PMDI) 2007-2023 - onde estão estabelecidas estratégias que visam o desenvolvimento do Estado.

\subsubsection{Lei mineira de inovação e Fiit}

Sancionada em 17 de janeiro de 2008, a Lei Mineira de Inovação ( $\left.n^{0} 17.348\right)$ dispõe sobre o incentivo à inovação tecnológica em Minas Gerais, buscan- 
do promover medidas de fomento à pesquisa científica e tecnológica, a capacitação e a competitividade no processo de desenvolvimento industrial do Estado.Um fator importante da lei é a criação do Fundo de Incentivo à Inovação Tecnológica (FIIT) que consiste em um instrumento de aceleração do processo inovativo (INSTITUTO INOVAÇÃO 2008).

O FIIT tem por objetivo a promoção e o desenvolvimento da inovação tecnológica no Estado de Minas Gerais através de programas e ações que objetivam (Decreto Estadual no 44.874, de 18/08/2008 - Regulamento do FIIT): i) estimular a pesquisa e o desenvolvimento de produtos e processos inovadores nas empresas sediadas no Estado de Minas Gerais; ii) dar suporte e apoio financeiro a projetos de pesquisa e desenvolvimento de produtos e processos inovadores nas EBTs e nas ICT-Privadas; iii) estimular a constituição de alianças estratégicas e o desenvolvimento de projetos de cooperação, envolvendo empresas mineiras e instituições públicas e de direito privado, sem fins lucrativos, voltadas para atividades de pesquisa e desenvolvimento, que objetivem a geração de produtos e processos inovadores.

\subsubsection{Sects}

Em sinergia com o Estado, a Secretaria de Estado de Ciência, Tecnologia e Ensino Superior (Sectes) elaborou o documento "Gestão Estratégica: Plano Diretor 2007-2011" com o intuito de formular políticas que sustentarão a gestão da Secretaria até 2011. Cabe ressaltar que a elaboração do referido documento levou em consideração os cenários e políticas contidos no PMDI 2007/2023 (Secretaria de Estado do Planejamento e Gestão 2007), incorporando-se a eles as especificidades de CT\&I e Ensino Superior.

Para a Sectes, o desafio se dá em desenvolver mecanismos que possam avaliar e disseminar os benefícios econômicos, sociais e ambientais existentes na C\&T. Só assim, os setores da economia reconhecerão CT\&I como área estratégica de desenvolvimento capaz de alavancar um país (SECTES 2007). Desta forma, em 2007 foi criado o Projeto Estruturador Rede de Inovação Tecnológica (RIT) que tem o objetivo de ampliar a capacidade de inovação tecnológica do setor produtivo mineiro, fomentando e articulando os diferentes agentes empresariais, governamentais, do setor acadêmico e da sociedade, dinamizando o sistema mineiro de inovação.

O projeto desenvolve cinco linhas de ação (SECTES 2010b): o apoio à criação, ampliação e gestão de habitats de inovação; a efetivação do arcabouço legal da inovação, que culminou na elaboração e promulgação da Lei Mineira de Inovação, com a criação do FIIT, e na criação e fortalecimento da Rede Mineira de Propriedade Intelectual; o suporte aos processos de inovação e empreendedorismo tecnológico; o fomento à cultura empreendedor; a articulação de parcerias e interações estratégicas, que envolve a interação universidade/ 
empresa/governo promovida pelo portal SIMI e a efetivação de parcerias para execução colaborativa de políticas públicas.

Um dos programas estabelecidos no RIT é o Programa de Incentivo à Inovação (PII), implementado pela Sectes e Sebrae-MG nas universidades mineiras, que tem extrema importância para a geração de spin-offs, intensificando a interação universidade-empresa.

Vale destacar que a Sectes é o órgão gestor da Fundação de Amparo à Pesquisa do Estado de Minas Gerais - Fapemig. A Fapemig é a única agência de fomento ao desenvolvimento científico e tecnológico de Minas Gerais e tem como finalidade a promoção de atividades de fomento e o apoio e incentivo à pesquisa científica e tecnológica no Estado. Além disso, é o agente executor e financeiro do FIIT.

\section{Linhas de financiamento de parques tecnológicos}

Neste capítulo são apresentadas as principais fontes de recursos utilizadas na consolidação de PqTs instalado no Estado de Minas Gerais. A apresentação foi dividida em dois itens: Recursos alocados pelo Governo Federal e Recursos Alocados pelo Estado de Minas Gerais.

\subsection{Recursos alocados pelo governo federal}

O Governo Federal possui três modalidades de apoio à implantação física de PqTs (ANPROTEC 2008): i) Não Reembolsável - recursos públicos viabilizados junto ao orçamento da união por emendas parlamentares. Além disso, agentes do Sistema de C\&T\&I tais como Finep e BNDES também oferecem apoio; ii) Financiamento: linhas de financiamento em condições especiais, operacionalizadas pelos agentes Finep, BNDES e outros agentes do sistema financeiro; e iii) Participação no capital de PqTs: instrumentos de participação no capital de PqTs, visando agregar recursos e, principalmente, expertise, networking e credibilidade, especialmente a partir do BNDES.

Desta forma, foi feito um levantamento das três fontes de recursos federais para PqTs: Emenda Parlamentar Federal, Finep e BNDES.

O mecanismo de emenda parlamentar consiste na principal estratégia adotada por Estados e Municípios para obtenção de recursos de investimento em projetos de PqTs (ANPROTEC 2008). A partir da Constituição Federal de 1988, o poder de emenda dos parlamentares foi ampliado, possibilitando alterações dos projetos do orçamento anual e das diretrizes orçamentárias. Neste contexto, as emendas parlamentares constituem-se como um instrumento para a alteração da proposta orçamentária elaborada pelo poder Executivo, permitindo que o Legislativo participe desse processo (Carvalho 2008). 
No que tange os recursos providos pela Finep, foi feita uma análise dos investimentos realizados pela financiadora, cujo período é de 2002 a 2008, através dos relatórios contidos no site da mesma. Verificou-se que a Finep possui apenas uma forma de fomento diretamente ligado a implantação de PqTs: Edital Verde Amarelo/Parques Tecnológicos.

O documento "Ciência, Tecnologia e Inovação para o Desenvolvimento Nacional: Plano de Ação 2007-2010” profere que:

a FINEP criou o Programa Pró-Parques, de crédito com juro zero, 100 meses para pagar, 2 anos de carência e sem garantias reais, destinado a entidades gestoras de parques tecnológicos credenciados pela FINEP e que será implementado a partir de 2008. (Secretaria de Estado do Planejamento e Gestão 2007)

Entretanto, é possível que esse programa não esteja ainda em funcionamento, tendo em vista informações solicitadas à fonte interna à FINEP, que apenas destacou o Edital Verde-Amarelo/Parques Tecnológicos.

Quanto ao BNDES (2010), os PqTs são financiados através de mecanismos de apoio à inovação e são divididos em recursos reembolsáveis (Capital Inovador, Inovação Tecnológica, Inovação Produção) e não reembolsáveis (BNDES Funtec).

Além dos instrumentos de financiamentos de PqTs apresentados, o MCT assegura, para 2010, uma chamada pública no valor de R $\$ 110$ milhões para serem investidos em PqTs, sendo que o valor para cada projeto irá variar de R\$ 5 a R\$ 30 milhões (França 2010).

\subsection{Recursos alocados pelo estado de Minas Gerais}

O Governo do Estado, em consonância com a política pública estadual de apoio à inovação, tem investido densamente na criação de PqTs mineiros. Segundo Sectes (2010a), o Estado tem a previsão de desembolsar cerca de R \$ 42 milhões no período de 2007-2010. Este montante irá beneficiar a construção e o provimento da infraestrutura para atração de investimentos intensivos em tecnologia e inovação tecnológica, a definição dos modelos de gestão e ocupação, assim como a atração de empreendimentos para os parques.

Por meio da RIT, atualmente, o governo mineiro apoia cinco parques: Belo Horizonte, Itajubá, Viçosa, Juiz de Fora e Lavras.

Um dos principais critérios utilizados pelo Estado para a seleção dos parques a serem apoiados é a sua ligação com universidades, devido à aproximação com a produção de conhecimento científico ser considerada um pré-requisito para um melhor desenvolvimento dos parques (Menezes 2010a). Os critérios, que 
podem ser visualizados no Quadro 1, estão descritos na Instrução Normativa $\mathrm{n}^{\circ}$ 01, de 05 de novembro de 2008, a qual disciplina o credenciamento para participação do Programa de Implantação e Consolidação de Parques e Polos Tecnológicos em Minas Gerais (PROPARQUE), constituído no Decreto Estadual $\mathrm{n}^{\circ} 42.368$, de 06 de fevereiro de 2002, conforme exposto abaixo. 
QUADRO O1.CRITÉRIOS PARA O CREDENCIAMENTO COMO PARTICIPANTE DO PROPARQUE

\begin{tabular}{|c|c|}
\hline \multicolumn{2}{|r|}{ CRITERIOS } \\
\hline $\mathbf{I}$ & $\begin{array}{l}\text { Demonstre a viabilidade técnica, econômica e financeira do empreendi- } \\
\text { mento, incluindo, se necessário, projetos associados e complementares } \\
\text { em relação às atividades principais do Parque; }\end{array}$ \\
\hline II & $\begin{array}{l}\text { Possua modelo de gestão adequado à realização de seus objetivos, o qual } \\
\text { deverá prever órgão técnico que tenha por finalidade zelar pelo cumpri- } \\
\text { mento do objeto social do Parque Tecnológico; }\end{array}$ \\
\hline III & $\begin{array}{l}\text { Comprove que dispõe, para desenvolver suas atividades, de recursos } \\
\text { próprios ou oriundos de instituições de fomento, financeiras ou de apoio } \\
\text { às atividades empresariais; }\end{array}$ \\
\hline IV & Tenha personalidade jurídica própria e objeto social específico; \\
\hline $\mathbf{V}$ & $\begin{array}{l}\text { Apresente projeto urbanístico-imobiliário para a implantação de empresas } \\
\text { inovadoras ou intensivas em conhecimento, instituições de pesquisa e } \\
\text { prestadoras de serviços ou de suporte à inovação tecnológica; }\end{array}$ \\
\hline VI & $\begin{array}{l}\text { Apresente projeto de planejamento que defina e avalie o perfil das ativida- } \\
\text { des do Parque, de acordo com as competências científicas e tecnológicas } \\
\text { das entidades locais e as vocações econômicas regionais; }\end{array}$ \\
\hline VII & $\begin{array}{l}\text { Estimule o surgimento, o desenvolvimento, a competitividade e o au- } \\
\text { mento da produtividade de empresas, no âmbito do Estado de Minas } \\
\text { Gerais, cujas atividades estejam fundadas no conhecimento e na inovação } \\
\text { tecnológica; }\end{array}$ \\
\hline VIII & $\begin{array}{l}\text { Incentive a interação e a sinergia entre empresas, instituições de pesqui- } \\
\text { sa, universidades, instituições prestadoras de serviços ou de suporte às } \\
\text { atividades intensivas em conhecimento e inovação tecnológica; }\end{array}$ \\
\hline \multicolumn{2}{|r|}{ CRITÉRIOS } \\
\hline IX & $\begin{array}{l}\text { Promova parcerias entre instituições públicas e privadas envolvidas com } \\
\text { a pesquisa científica, a inovação tecnológica inerente aos serviços e a infra } \\
\text { estrutura tecnológica de apoio à inovação; }\end{array}$ \\
\hline $\mathbf{X}$ & $\begin{array}{l}\text { Apoie atividades de pesquisa, desenvolvimento e de engenharia comple- } \\
\text { mentares ao desenvolvimento dos arranjos produtivos locais; }\end{array}$ \\
\hline $\mathbf{X I}$ & $\begin{array}{l}\text { Propicie o desenvolvimento do Estado de Minas Gerais, por meio da } \\
\text { atração de investimentos em atividades intensivas em conhecimento e } \\
\text { inovação tecnológica. }\end{array}$ \\
\hline
\end{tabular}

FONTE: Adaptado de Minas Gerais (2008). 
O governo de Minas Gerais possui recurso para a implantação de três parques (Belo Horizonte, Itajubá e Viçosa) que serão finalizados (edificações, licenciamento ambiental, modelo jurídico definido) até 2010. Segundo Sectes (2010b: 1), "o Governo de Minas vai entregar, além da estrutura física, a estrutura logística da gestão e governança dos parques”. Para os outros dois parques, a proposta é de alocação de recursos para estudo urbanístico e licenciamento ambiental. Na Tabela 1 estão especificados todos os recursos alocados até o final de 2010 por PqT apoiado por Minas.

TABELA 01 . RECURSOS ALOCADOS EM PQTS MINEIROS (EM R\$ MILHÕES)

\begin{tabular}{c|c|c|c|c|c|c}
\hline \multicolumn{7}{c}{ RECURSOS ALOCADOS POR PARQUE } \\
\hline Parque & $\begin{array}{c}\text { Belo } \\
\text { Horizonte }\end{array}$ & Itajubá & Viçosa & $\begin{array}{c}\text { Juiz } \\
\text { de } \\
\text { Fora }\end{array}$ & Lavras & TOTAL \\
\hline $\begin{array}{c}\text { Governo } \\
\text { de } \\
\text { Minas }\end{array}$ & 24,60 & 10,00 & 9,30 & 3,15 & 1,56 & $\mathbf{4 8 , 6 1}$ \\
\hline Outros & 6,40 & 16,00 & 0,50 & 6,20 & 0,54 & $\mathbf{2 9 , 6 4}$ \\
\hline Total & $\mathbf{3 1 , 0 0}$ & $\mathbf{2 6 , 0 0}$ & $\mathbf{9 , 8 0}$ & $\mathbf{9 , 3 5}$ & $\mathbf{2 , 1 0}$ & $\mathbf{7 8 , 2 5}$ \\
\hline
\end{tabular}

FONTE: Elaborada pela autora baseada em informações de Sectes (2010c).

Mencionados os critérios para a inserção no PROPARQUE, o apoio financeiro disponibilizado pelo Estado se configura através das seguintes formas: "dinheiro induzido" (Convênio), BDMG e emenda parlamentar estadual.

O recurso investido através de "dinheiro induzido", em sua maior parte, decorre do Tesouro do Estado e constitui a principal fonte. A origem do recurso é arrecadação estadual, sendo seu principal montante proveniente de ICMS.

No que tange o mecanismo de emenda parlamentar estadual, Bakô (2010) diz que também constitui uma fonte de recurso para os parques, porém não garante o empreendimento e é utilizada como complementação dos recursos. É importante destacar que, para ser aprovada, esta forma deve estar em acordo com a política do Estado.

Através do BDMG, PqTs alocados no Estado de Minas Gerais podem obter recurso, porém sua disponibilização depende da personalidade jurídica do parque, assim como sua localização. A “definição desses dados é indispensável para verificarmos quem será o proponente do crédito e quais as condições específicas das fontes de recursos." (Fiorini 2010). Apesar de possuir linhas diretas aos parques, o BDMG é mais utilizado pelas empresas instaladas nos PqTs devido às condições especiais que apresenta.

Na Tabela 2 são apresentadas as fontes de recursos e valores dos PqTs apoiados pelo Estado de Minas Gerais. Cabe destacar que os dados são relativos 
ao período de 2002 a 2010. Como pode ser visualizado, o maior aporte financeiro dos PqTs inseridos em Minas provém de recursos do Governo do Estado. Ademais, destaca-se a significante quantia provida por emendas parlamentares.

TABELA 2 . FONTES DE RECURSOS X VALORES RELATIVOS AO PERÍODO DE 2002 A 2010 (EM R\$)

\begin{tabular}{c|c|c|c|c|c|c}
\hline \multirow{2}{*}{ Fontes } & \multicolumn{3}{c|}{ RECURSO FEDERAL } & \multicolumn{2}{c}{ RECURSO ESTADUAL } \\
\cline { 2 - 7 } & $\begin{array}{c}\text { Emenda } \\
\text { Parlamentar }\end{array}$ & FINEP & BNDES & $\begin{array}{c}\text { Emenda } \\
\text { Parlamentar }\end{array}$ & $\begin{array}{c}\text { Dinheiro } \\
\text { Induzido }\end{array}$ & BDMG \\
\hline $\begin{array}{c}\text { Belo } \\
\text { Horizonte }\end{array}$ & -- & $5.893 .420,00$ & - & -- & $20.933 .523,92$ & - \\
\hline $\begin{array}{c}\text { Itajubá } \\
\text { Viçosa }\end{array}$ & $1.400000,00$ & $1.249 .733,00$ & - & -- & $5.012 .000,00$ & - \\
\hline $\begin{array}{c}\text { Juiz de } \\
\text { Fora }\end{array}$ & $6.200 .000,00$ & - & - & $1.500 .000,00$ & $150.000,00$ & - \\
\hline Lavras & -- & $1.717 .719,00$ & - & -- & $60.000,00$ & - \\
\hline TOTAL & $7.760 .000,00$ & $8.860 .872,00$ & - & $1.500 .000,00$ & $32.090 .523,92$ & - \\
\hline
\end{tabular}

FONTE: Elaborada pela autora baseada em informações obtidas no CRITT/UFJF e de Menezes (2010b).

No que diz respeito aos recursos aportados pela Finep, de acordo com a Tabela 2, os recursos foram obtidos pelo Governo do Estado de Minas Gerais que submeteu um projeto ao Edital Finep Estruturante. Os recursos obtidos a partir desse edital foram alocados aos parques pelo Estado de Minas.

Vale ressaltar que em 2001 e 2002, a Fapemig fez duas chamadas públicas para o Edital "Apoio Complementar à Formação de Parques Tecnológicos", sendo obtido um total de 12 projetos.

Segundo Bakô (2010), a chamada não se preocupou em predeterminar os setores econômicos nem a natureza tecnológica das propostas enviadas ao governo estadual. Desta forma muitas propostas possuíam conteúdo que não apresentavam grande impacto em termos de indução do desenvolvimento tecnológico regional. Além disso, não havia capacidade de implementação financeira e de know how por parte do Estado, sendo necessário um processo de seleção dos parques, cujos critérios estão apresentados no Quadro 1. A partir dessa experiência, o Governo Estadual optou por não mais utilizar de Chamada Pública, considerando a criação de PqTs como política pública que deve ser realizada de forma estratégica e pontual.

Até então, as fontes de recursos especificadas são as utilizadas para a implan- 
tação do parque. Por outro lado, a operacionalização desse empreendimento irá depender do modelo jurídico adotado, assim como do modelo de gestão (Nepomuceno 2010). Contudo, são apresentadas algumas possíveis fontes de recursos para operacionalização de um PqT (Figlioli 2007): investimento direto das universidades, locação dos prédios, taxas administrativas, aluguel dos espaços nas incubadoras, recursos de projetos, venda de terrenos ou permuta por área construída, venda de edifícios construídos pelo parque, Dividendos de participação em Sociedade para Propósito Específico (SPE) - parceria com investidores para implantar empreendimentos no parque, serviços de gestão, receita de serviços de manutenção do condomínio. Essas informações foram elaboradas com base em um estudo sobre financiamentos de PqTs, no qual a pesquisa foi feita nos seguintes parques: Tecnopuc, Polo de Informática de São Leopoldo e Sapiens Parque, instalados no Brasil; Biocant Park e Taguspark, instalados em Portugal; e Parque Tecnológico de Cartuja 93, instalado na Espanha. Destarte, essas informações devem ser utilizadas apenas como base para desdobramentos futuros de pesquisa.

Vale destacar a importância de atrair empreendimentos âncoras para serem inseridos nos parques, ou seja, as grandes empresas que atraem uma cadeia de pequenas e médias empresas, normalmente suas fornecedoras. Além das possíveis fontes de recursos apresentadas, Bakô (2010) destaca que o fator primordial para o funcionamento de um parque é a constituição de parcerias estratégicas para o empreendimento. Dessas parcerias, destacam-se: Prefeituras; Governo do Estado; Instituições Públicas, como SEBRAE; Entidades Empresarias; Instituições de Pesquisa; Empresas Emergentes.

\section{Conclusão}

O estímulo à interação entre empresas e universidades faz-se imprescindível no atual cenário econômico, baseado em conhecimento, tendo em vista a necessidade de geração de empresas inovadoras capazes de aumentar a competitividade brasileira frente às outras economias.

Para que a inovação cumpra seu papel nessa nova economia, é imprescindível desenvolver o SNI do Brasil, incorporando seus aspectos culturais e históricos. A formatação de um sistema pressupõe um conjunto de elementos interconectados de forma a permitir a concepção de uma totalidade, o que implica que o SNI brasileiro deva promover ações que estimulem e viabilizem a interação universidade-empresa-governo.

Na realidade brasileira, o governo deve atuar como protagonista no processo de integração entre a infraestrutura científico-tecnológica e o setor produtivo, o que se concretiza através da elaboração de políticas públicas de incentivo à transferência dos conhecimentos desenvolvidos no âmbito acadêmico para a esfera empresarial, além da oferta de recurso financeiro para o funciona- 
mento do SNI.

Nesse novo contexto, também conhecido como economia do aprendizado, as universidades, principalmente as públicas, além de pesquisa base, passam a desenvolver pesquisa aplicada que é comercializada por meio da transferência de tecnologia para o setor produtivo ou da geração de spin-offs. Desta forma, assumem a missão de empreendedoras e se configuram como principais desenvolvedoras de habitats de inovação - incubadoras de empresas e PqTs.

Mais do que um empreendimento de grande porte, o PqT se consolida como o principal modelo de habitat de inovação, que se configura como uma política pública capaz de gerar sinergias além de permitir maior competitividade empresarial, maior demanda por empregos que requerem recursos humanos altamente capacitados e maior arrecadação de impostos. Em suma, contribui para o desenvolvimento regional que gera um "ciclo virtuoso" na economia.

Para que este empreendimento seja constituído, exige-se vultoso aporte financeiro para sua implantação e contínua operação, pois além de uma estrutura especialmente planejada, este empreendimento necessita de recursos humanos altamente capacitados. Desta forma, a obtenção dos recursos financeiros pode ser considerada um dos principais entraves à realização desse empreendimento.

Por isso, este trabalho pesquisou as fontes de apoio financeiro existentes no Brasil para a implantação de PqTs no Estado de Minas Gerais, são elas: emenda parlamentar federal e estadual; Finep, via Edital Verde Amarelo/ Parques Tecnológicos; Recurso do Tesouro do Estado de Minas Gerais, via demanda induzida; e BNDES e BDMG, ainda que esses últimos sejam mais focados nas empresas instaladas nos parques.

Das fontes apresentadas, atualmente, Recurso do Tesouro do Estado de Minas Gerais consiste na principal fonte de recursos para a implantação de PqTs no Estado de Minas Gerais. Entretanto, cabe destacar o significativo aporte financeiro provido de emendas parlamentares. Isso demonstra que a viabilização de tal empreendimento está muita atrelada às questões políticas, o que dá margem à possibilidade de conflitos e disputas pelos recursos disponíveis.

No que tange à sustentabilidade do empreendimento, os recursos para sua manutenção dependem do modelo de negócio adotado por cada parque, porém é relevante levantar alguns questionamentos: "como manter a sustentabilidade dos Parques em Minas Gerais?”, “o Estado deve investir na manutenção dos PqTs?”.

Para minimizar a participação do Estado na manutenção financeira dos PqTs, uma alternativa a ser considerada é a criação de medidas de incentivo à consolidação de parcerias estratégicas e à atração de empreendimentos âncora concomitantemente ao processo de implantação desses parques. Ademais, tendo em vista o caráter ainda embrionário da experiência brasileira nas 
atividades de PqTs, fazem-se pertinentes estudos acerca da sustentabilidade dos modelos existentes.

A presente pesquisa mostrou que o tema PqT ainda é muito recente no Brasil, principalmente quando se trata dos recursos envolvidos. Apesar do grande número de projetos de parques a serem implantados no País, ainda não foi detectado um planejamento de quais serão apoiados, o que demonstra desorganização dos investimentos realizados, uma vez que determinados projetos receberam recursos e estes empreendimentos não foram implementados.

O Brasil possui políticas públicas de apoio à inovação, porém, quanto aos PqTs o País não apresenta um programa que permite a captação de recursos nas diferentes fases de desenvolvimento do empreendimento, fazendo com que os investimentos públicos existentes não sejam suficientes para a obtenção dos resultados almejados, tampouco para atrair os investimentos privados.

Diante do cenário apresentado, talvez seja necessário criar uma estrutura de apoio aos PqTs, interligando as diferentes esferas governamentais (federal, estadual e municipal) com o intuito de garantir a continuidade dos investimentos, além de evitar o desperdício de dinheiro público.

Contudo, para que esse tipo de empreendimento promova o efeito ativo e retroativo do esforço coordenado dos diferentes agentes envolvidos, é necessário que os PqTs sejam tratados como política pública, em todas as esferas governamentais, indutora e fiscalizadora da cooperação entre instituições de ensino e empresas, com foco nas áreas estratégicas de desenvolvimento do País. Para tanto, os estudos acerca dessa temática devem ser aprofundados com o objetivo de desenvolver um programa brasileiro de apoio a parques, no qual devem estar previstos os recursos financeiros, o acompanhamento da aplicação desses recursos e os resultados alcançados pelos parques. Destarte, é relevante destacar a continuidade de estudos com o intuito de avaliar o impacto econômico das políticas públicas relativas aos PqTs.

\section{Referências}

ABDI/ ANPROTEC (2007). Parques Tecnológicos no Brasil: Estudo, Análise e Proposição. Brasília: Consenso Editora Gráfica.

ANPROTEC (2008). Portfólio de Parques Tecnológicos no Brasil. Brasília.

BAKÔ, Anna Flávia (2010). Entrevista com a gerente-adjunta da Rede de Inovação Tecnológica da Sectes. Belo Horizonte.

BERNARDES, A. \& ALBUQUERQUE, E. (2003). "Cross-over, thresholds, and interactions between science and technology: lessons for less-developed countries”. Research Policy 32: 865-885.

BNDES (2010). Financiamento a Parques Tecnológicos. [mensagem pessoal] Mensagem recebida por: <inaiaracoser@bol.com.br>. 
BRASIL (2004). "Lei n. 10973, de 2 de dezembro de 2004. Dispõe sobre incentivos à inovação e à pesquisa científica e tecnológica no ambiente produtivo e dá outras providências" URL: http://www.planalto.gov.br/ccivil_03/_ato20042006/2004/Lei/L10.973.htm. Acesso em 01 de março de 2010.

CARVALHO, Kildare Gonçalves (2008). “Processo Legislativo”. In CARVALHO, Kildare Gonçalves. Direito Constitucional. 14. ed. Belo Horizonte: Del Rey.

CASSIOLATO, José Eduardo \& LASTRES, Helena Maria Martins. (200o). "Sistemas de Inovação: Políticas e Perspectivas”. Parcerias Estratégicas 08: 237-255.

ETZKOWITZ, Henry. \& LEYDESDORFF, Loet. (1997). Universities and the Global Knowledge Economy: A Triple Helix of University- Industry-Government Relations. London: Cassell Academic.

ETZKOWITZ, Henry., LEYDESDORFF, Loet. (2000). “The dynamics of innovation: from national systems and model 2 to a triple helix of university-industrygovernment relations". Research Policy 29: 109-123.

ETZKOWITZ, Henry (1998). “The Norms of Entrepreneurial Science: Cognitive Effects of the New University-Industry Linkages”. Research Policy 27: 823-833.

ETZKOWITZ, Henry. (2002). "The Triple Helix of University - Industry - Government: Implications for Policy and Evaluation”. In SISTER, Working Paper 2002-11.

FIGLIOLI, Aline. (2007). Perspectivas de Financiamento de Parques Tecnológicos: um estudo comparativo. Ribeirão Preto.

FINEP (2006). "Esclarecimentos sobre o programa de subvenção econômica à inovação" URL: http://www.finep.gov.br/imprensa/noticia.asp?cod_noticia=1022. Acesso em: 04 fevereiro de 2010.

FINEP (2010). “O que são os Fundos de C\&T". URL: http://www.finep.gov.br/ fundos_setoriais/fundos_setoriais_ini.asp?codSessaoFundos=1. Acesso em: 04 de fevereiro de 2010.

FINEP (2002 a 2008). “Relatórios de Gestão” URL: http://www.finep.gov.br/numeros_finep/relatorios_ini.asp. Acesso em: 02 de fevereiro de 2010.

FIORINI, Sérgio Luís Costa. (2010). Financiamento a Parques Tecnológicos. [mensagem pessoal] Mensagem recebida por: <inaiaracoser@bol.com.br>.

FRANÇA, Martha San Juan. (2010). "Inovação precisa de pacotes de incentivos”. Brasil Econômico URL: http://www.tramaweb.com.br/upload/midia/ gde/11052010_BE2_2010520154834.jpg. Acesso em: 21 de maio de 2010.

FREEMAN, C.\& PEREZ, C. (1988). "Structural crises of adjustment business cycles and investment behaviour”. In DOSI, G. et al. (ed.). Technical change and economic theory. London: Pinter.

HILPERT, U. RUFFIEUX, B. (1991). "Innovation, politics and regional development: technology parks and regional participation in high tech in France and West Germany". In (ed.), Regional innovation and decentralization : high tech industry and government policy. London; New York: Routledge.

HU, A. G. (2007). "Technology parks and regional economic growth in China”. Research Policy 36: 76-87. 
ICHIKAWA, E. Y. \& SANTOS, L. W. (2001). “Inovação Tecnológica e Desenvolvimento: A Relação Universidade/Empresa Vista sob o Paradigma da Hélice Tríplice”. In IEL-PR. Monografias Premiadas no $2^{\circ}$ Concurso de Monografias sobre a Relação Universidade/Empresa. Curitiba: IPARDES, pp. 221-244.

INSTITUTO INOVAÇÃO (2008). "Lei Mineira de Inovação é Sancionada” URL: http://www.institutoinovacao.com.br/internas/noticia/idioma/1/129. Acesso em: 08 de março de 2010.

INTERNATIONAL ASSOCIATION OF SCIENCE PARKS - IASP (2002). "About Science and Technology Parks - Definitions” URL: http://www.iasp.ws/publico/ index.jsp?enl=2. Acesso em: 24 de outubro de 2009.

JOHNSON, Björn \& LUNDVALL, B.-Å. (2005). "Promovendo sistemas de inovação como resposta à economia do aprendizado crescentemente globalizada". In LASTRES, Helena M. M. \& CASSIOLATO, José E. \& ARROIO, Ana (orgs). Conhecimento, Sistemas de Inovação e Desenvolvimento. Rio de Janeiro: Editora UFRJ; Contraponto, pp. 83-130.

JOWITT, Anthony (1991). "Science parks, academic research and economic regeneration. In Regional innovation and decentralization: high tech industry and government policy. London; New York: Routledge.

LACAVE, Michel. (1996). "Is the science parks movement victim of its success? A plea for reengineering”. In World Conference on Science Parks, 5, 1996, Rio de Janeiro. Proceedings... Rio de Janeiro: ANPROTEC p. 342-346

LEYDESDORFF, Loet \& ETZKOWITZ, Henry (1998). “The Triple Helix as a Model for Innovation Studies". (Conference Report), Science \& Public Policy 25(3): 195-203.

LINDELÖF, Peter \& LÖFSTEN, Hans (2002). "Growth, management and financing of new tecnology-based firms - assessing value-added contributions of firms located on and off Science Parks". Omega 30: 143-154.

LÖFSTEN, Hans; LINDELÖF, Peter. (2002). "Science Parks and the growth of new technology-based firms-academic-industry links, innovation and markets". Research Policy 31: 859-876.

LUNDVALL, B.A. (1998). “Innovation as an interactive process: from user-producer interaction to the national system of innovation”. In DOSI, G. et al. (eds.). Technical change and economic theory. London: Pinter Publishers.

LUNDVALL, B.-Å. (1992). National systems of innovation: towards a theory of innovation and interactive learning. London: Pinter.

MARTINS, Maximiano. (1996). "The dynamizing role in regional development of science \& technology parks". In World Conference on Science Parks, 5, 1996, Rio de Janeiro. Proceedings... Rio de Janeiro: ANPROTEC. p.244-258

MENEZES, Felipe. (2010a). Entrevista com o especialista em políticas públicas da Sectes. Belo Horizonte.

MENEZES, Felipe. (2010b). Tabela de Repasses. [mensagem pessoal] Mensagem recebida por: <inaiaracoser@bol.com.br>.

MINAS GERAIS (2008). Instrução normativa $n^{\circ}$ o1, de 05 de novembro de 2008. Disciplina o credenciamento para participação no programa de implantação e consolidação de parques e pólos tecnológicos em minas gerais, através do grupo 
coordenador. Secretaria de Estado de Ciência, Tecnologia e Ensino Superior, Belo Horizonte.

MORAIS, Ednalva F. C.\& MATTOS, J. Fernando\& GASTAL, Cláudio (2006). Mecanismos de Inovação e Competitividade. Brasília: MBC.

MORAIS, José Mauro de (2008). Uma Avaliação de Programas de Apoio Financeiro à Inovação Tecnológica com Base nos Fundos Setoriais e na Lei de Inovação. In IPEA. Políticas de Incentivo à Inovação Tecnológica. Brasília: Livraria, pp. 67-105.

MOTHE, J.\& PAQUET, Gilles (1998). National Innovation Systems, =Real Economies' and Instituted Processes. Small Business Economics 11: 101-111.

NELSON, R (1988). Institutions supporting technical change in the US. In DOSI, G. et al. (ed.). Technical change and economy theory. London: Pinter, pp. 312-329.

NEPOMUCENO, Paulo (2010). Entrevista com o Secretário de Desenvolvimento Tecnológico da UFJF e coordenador do projeto Parque Tecnológico de Juiz de Fora. Juiz de Fora.

OECD (2009). National Innovation Systems. 1997. URL: www.oecd.org/dataoecd/35/56/210733.pdf. Acesso em: 16 de dezembro de 2009

PACHECO, C. A. (2005). Políticas públicas, intereses y articulación política: cómo se gestaron las recientes reformas al sistema de ciencia y tecnología en Brasil. Santiago: Cepal, Serie Políticas Sociales.

SECRETARIA DE ESTADO DO PLANEJAMENTO E GESTÃO (2007). Plano Mineiro de Desenvolvimento Integrado (PMDI) 2007-2023. Belo Horizonte, p. 52.

SECTES (2007). Gestão Estratégica: Plano Diretor 2007-2011.

SECTES (2010a). “Implantação dos parques tecnológicos” URL: http://www.tecnologia.mg.gov.br/index.php/programas/projetos-estruturadores/rit/parquestecnologicos. Acessoem: 18 de maio de 2010.

SECTES (2010b). "Livro com resultados do programa de incentivo à inovação na UFMG é lançado em Belo Horizonte”. URL: http://www.tecnologia.mg.gov.br/ index.php/todas-as-noticias/21-noticia-destaque/143-livro-com-resultadosdo-programa-de-incentivo-a-inovacao-na-ufmg-e-lancado-em-belo-horizonte. Acesso em: 15 de março de 2010.

SECTES (2010c). "Parque Tecnológico de Itajubá recebe novos investimentos do Governo de Minas”. URL: http://www.tecnologia.mg.gov.br/index.php/ noticias/206-parque-tecnologico-de-itajuba-recebe-novos-investimentos-dogoverno-de-minas. Acessado em 18 maio 2010.

SIEGEL, D. S. \& WRIGHT, M \& LOCKET, A. (2007). “The rise of entrepreneurial activity at universities: organizational and societal implications". Industrial and Corporate Change 16: 489-504.

SIEGEL, D. S.; WESTHEAD, Paul \& WRIGHT, M. (2003). "Science Parks and the Performance of New Technology-Based Firms: A Review of Recent U.K. Evidence and an Agenda for Future Research”. Small Business Economics 20: 177-184. 
STEINER, J. E. \& CASSIM, M. B. \&ROBAZZI, A. C (2008). "Parques Tecnológicos: Ambientes de Inovação”. URL: <http://www.iea.usp.br/iea/textos/steinercassimrobazziparquestec.pdf $>$. Acesso em: 10 de setembro de 2009.

ULLAH, F.; TAYLOR, P (2005). The impact of Science Park and Incubator Location on the Finance Opportunities of Technology-Based Small Firms. In DURING W., OAKEY R. \& KAUSER S. New Technology-Based Fimrs in the New Millennium Volume IV. Elsevier, pp. 249-261.

YANG, Chih-Hai \& MOTOHASHI, Kazuyuki \& CHEN, Jong-Rong (2009). Are new technology-based firms located on science parks really more innovative? Evidence from Taiwan. Research Policy 38: 77-85.

ZEN, A. C. \& HAUSER, G. \& VIEIRA, C. R. B (2004). Parques Tecnológicos: três modelos internacionais e a perspectiva para o movimento no Brasil. In: Seminário ANPROTEC, 16, Porto de Galinhas. Anais... Porto de Galinhas: ANPROTEC.

ZOUAIN, D. M. (2003). Parques Tecnológicos: Propondo um Modelo Conceitual para Regiões Urbanas - O Parque Tecnológico de São Paulo. 248 f.

Recebido em: 16 de agosto de 2010

Primeira resposta em: 19 de outubro de 2010

Aceite em: 09 de novembro de 2010 